\title{
FOURIER SERIES OF FUNCTIONS WHOSE HANKEL TRANSFORM IS SUPPORTED ON $[0,1]$
}

\author{
JUAN L. VARONA*
}

\begin{abstract}
Let $J_{\mu}$ denote the Bessel function of order $\mu$. For $\alpha>-1$, the system $x^{-\alpha / 2-1 / 2} J_{\alpha+2 n+1}\left(x^{1 / 2}\right), n=0,1,2, \ldots$ is orthogonal on $L^{2}\left((0, \infty), x^{\alpha} d x\right)$. In this paper we study the mean convergence of Fourier series with respect to this system for functions whose Hankel transform is supported on $[0,1]$.
\end{abstract}

1991 Mathematics Subject Classification: Primary 42C10; Secondary 44A05.

Key words and phrases: Bessel functions, Fourier series, Hankel transform, $A_{p}$-theory.

\section{$\S 1$. Introduction.}

Let $J_{\mu}(x)$ stand for the Bessel function of order $\mu$ and $\left\{P_{n}^{(\alpha, \beta)}(x)\right\}_{n=0}^{\infty}$ for the Jacobi polynomials (see [15] and Ch. VII and X in [5]). It is well known that the Jacobi polynomials are orthogonal on $(-1,1)$ with respect to the weight $(1-x)^{\alpha}(1+x)^{\beta}, \alpha, \beta>-1$, and the Bessel functions satisfy the orthogonality relation

$$
\int_{0}^{\infty} J_{\alpha+2 n+1}(x) J_{\alpha+2 m+1}(x) \frac{d x}{x}=\frac{\delta_{n m}}{2(2 n+\alpha+1)}, \quad n, m=0,1,2, \ldots \quad(\alpha>-1) .
$$

If we denote

$$
j_{n}^{\alpha}(x)=\sqrt{\alpha+2 n+1} J_{\alpha+2 n+1}(\sqrt{x}) x^{-\alpha / 2-1 / 2}, \quad n=0,1,2, \ldots
$$

then the system $\left\{j_{n}^{\alpha}\right\}_{n=0}^{\infty}$ is orthonormal on $L^{2}\left(x^{\alpha}\right)=L^{2}\left((0, \infty), x^{\alpha} d x\right)$.

The Bessel functions and the Jacobi polynomials are related by the formula (see [6])

$$
\int_{0}^{\infty} J_{\alpha+2 n+1}(t) J_{\alpha}(x t) d t=x^{\alpha} P_{n}^{(\alpha, 0)}\left(1-2 x^{2}\right) \chi_{[0,1]}(x) .
$$

Following [4] we define the Hankel transform $\mathcal{H}_{\alpha}$ of order $\alpha>-1$ to be the integral operator

$$
\mathcal{H}_{\alpha}(f, x)=\frac{x^{-\alpha / 2}}{2} \int_{0}^{\infty} f(t) J_{\alpha}(\sqrt{x t}) t^{\alpha / 2} d t, \quad x>0,
$$

for suitable functions $f$.

This means that

$$
\mathcal{H}_{\alpha}\left(j_{n}^{\alpha}, x\right)=\sqrt{\alpha+2 n+1} P_{n}^{(\alpha, 0)}(1-2 x) \chi_{[0,1]}(x)
$$

* The author has been supported in part by DGICYT Grant PB89-0181-C02-02. PAPER PUBLISHED IN: Constr. Approx. 10 (1994), 65-75. 
and therefore $\operatorname{supp}\left(\mathcal{H}_{\alpha}\left(j_{n}^{\alpha}\right)\right) \subseteq[0,1]$.

We consider the partial sums of the Fourier series with respect to the system $\left\{j_{n}^{\alpha}\right\}_{n=0}^{\infty}$ :

$$
S_{n}(f, x)=\sum_{k=0}^{n} c_{k}(f) j_{k}^{\alpha}(x), \quad c_{k}(f)=\int_{0}^{\infty} f(t) j_{k}^{\alpha}(t) t^{\alpha} d t
$$

They can be written as

$$
S_{n}(f, x)=\int_{0}^{\infty} f(t) K_{n}(x, t) t^{\alpha} d t, \quad \text { where } \quad K_{n}(x, t)=\sum_{k=0}^{n} j_{k}^{\alpha}(x) j_{k}^{\alpha}(t)
$$

Series of this kind are a particular case of series $\sum_{n \geq 0} a_{n} J_{\alpha+n}$, which are usually called Neumann series. A study of their pointwise convergence can be found in [16] and [17].

The main aim in this paper is to study the convergence of $S_{n} f$ in the $L^{p}\left(x^{\alpha}\right)$-norm. This involves two problems:

a) To obtain uniform boundedness of the operators $S_{n} f$ in $L^{p}\left(x^{\alpha}\right)$.

b) To find the subspace of $L^{p}\left(x^{\alpha}\right)$ consisting of the functions $f$ which can be approximated in the $L^{p}\left(x^{\alpha}\right)$-norm by its Fourier series, that is, to describe the space

$$
B_{p, \alpha}=\overline{\operatorname{span}}\left\{j_{n}^{\alpha}(x)\right\}_{n=0}^{\infty} \quad\left(\text { closure in } L^{p}\left(x^{\alpha}\right)\right) .
$$

In order to solve a) the kernel $K_{n}$ is decomposed in a suitable way which reduces the problem to show the boundedness of the Hilbert transform with weights, and hence some estimates for the Bessel functions and some results on $A_{p}$ theory are needed. Some of these ideas have been used in the literature (see [1], [8], [9], [10], [12], [13]).

Regarding to b), looking at (3) we only need to deal with functions with Hankel transform supported on $[0,1]$. This leads us to consider, in a natural way, the analogous of the disc multiplier for the Hankel transform, i. e., the operator $M_{\alpha}$ defined by

$$
\mathcal{H}_{\alpha}\left(M_{\alpha} f, x\right)=\mathcal{H}_{\alpha}(f, x) \chi_{[0,1]}(x)
$$

Our problem of expanding a function whose Hankel transform is supported on $[0,1]$ with respect to an orthogonal system is in some sense similar to expanding a function whose Fourier transform is supported on $[-1,1]$, which has been treated in [1] using as an orthogonal system the spherical Bessel functions $\sqrt{\frac{\pi}{2 x}} J_{n+1 / 2}(x)$. The method we use to establish our results is easier than the one in [1]. Our development in $\S 2$ can be adapted to simplify some of the proofs in [1] and [2].

The paper is organized as follows: In section $\S 2$, we solve problem a). In section $\S 3$ we prove that the operator $M_{\alpha}$, defined for suitable functions $f$, can be extended from $L^{p}\left(x^{\alpha}\right)$ into itself and it turns out to be the projection operator. This allows us to study problem b) obtaining, in section $\S 4$, a characterization of $B_{p, \alpha}$ in terms of $M_{\alpha}$ and solving the convergence of $S_{n} f$ to $f$ in the $L^{p}\left(x^{\alpha}\right)$-norm. 


\section{$\S 2$. Uniform boundedness of the partial sums.}

In what follows, $\alpha \geq-\frac{1}{2}$ and $p_{0}=\frac{4(\alpha+1)}{2 \alpha+3}, p_{1}=\frac{4(\alpha+1)}{2 \alpha+1}$. From the well known estimates (see [5] or [15])

$$
J_{\mu}(x)=\frac{x^{\mu}}{2^{\mu} \Gamma(\mu+1)}+O\left(x^{\mu+2}\right), \quad x \rightarrow 0+
$$

and

$$
J_{\mu}(x)=\sqrt{\frac{2}{\pi x}}\left[\cos \left(x-\frac{\mu \pi}{2}-\frac{\pi}{4}\right)+O\left(x^{-1}\right)\right], \quad x \rightarrow \infty
$$

it follows

$$
\left|J_{\alpha}(x)\right| \leq C_{\alpha} x^{\alpha}, \quad x \in(0, \infty)
$$

and

$$
\left|J_{\alpha}(x)\right| \leq C_{\alpha} x^{-1 / 2}, \quad x \in(0, \infty)
$$

Given $p \in(1, \infty)$ and a fixed interval $(a, b)$, a weight $w$ is said to belong to the $A_{p}(a, b)$ class if

$$
\left(\int_{I} w(x) d x\right)\left(\int_{I} w(x)^{-1 /(p-1)} d x\right)^{p-1} \leq C|I|^{p}
$$

for every interval $I \subseteq(a, b)$, with $C$ independent of $I$. An important application of $A_{p}$ theory lies in its relation with the boundedness of the Hilbert transform

$$
H(f, x)=\int_{a}^{b} \frac{f(t)}{x-t} d t
$$

Indeed, in [11] (see also [7] for further information) it is proved that

$$
H: L^{p}((a, b), w) \longrightarrow L^{p}((a, b), w) \text { bounded } \Longleftrightarrow w \in A_{p}(a, b) .
$$

Besides, the norm of the Hilbert transform operator and the constant in the $A_{p}$ definition depend only one on each other. This allows us to use the uniform $A_{p}$ theory in a similar way to [10]. Let us suppose that a family of weights $\left\{w_{n}\right\}_{n=0}^{\infty}$ defined in the same interval $(a, b)$ satisfies the $A_{p}$ condition with the same constant $C$ (in this case we will say that $w_{n} \in A_{p}(a, b)$ uniformly). Then, the Hilbert transform $H$ is uniformly bounded from $L^{p}\left((a, b), w_{n}\right)$ into itself, that is, with constant independent of $n$.

It is well known

$$
x^{\beta} \in A_{p}(0,1) \Longleftrightarrow x^{\beta} \in A_{p}(1, \infty) \Longleftrightarrow x^{\beta} \in A_{p}(0, \infty) \Longleftrightarrow-1<\beta<p-1 .
$$

Moreover, by making the change of variable $x=r_{n} z$ we easily obtain that, if $r_{n} \searrow 0$, then

$$
\left(|x|+r_{n}\right)^{\beta} \in A_{p}(-1,1) \text { uniformly } \Longleftrightarrow-1<\beta<p-1 \text {. }
$$


By using (8), it is easy to prove that, if $\alpha \geq-1 / 2$ and $p_{0}<p<p_{1}$, then

$$
x^{\alpha-\alpha p / 2+p / 4} \in A_{p}(0, \infty) \quad \text { and } \quad x^{\alpha-\alpha p / 2-p / 4} \in A_{p}(0, \infty) .
$$

The following result will be used later.

Lemma 1. Let $\alpha \geq-1 / 2, \max \left\{\frac{4}{3}, p_{0}\right\}<p<\min \left\{4, p_{1}\right\}$ and $0<s_{n} \nearrow \infty$. Then

$$
x^{\alpha-\alpha p / 2+p / 8}\left(\left|x^{1 / 2}-s_{n}\right|+s_{n}^{1 / 3}\right)^{p / 4} \in A_{p}(0, \infty) \text { uniformly }
$$

and

$$
x^{\alpha-\alpha p / 2-p / 8}\left(\left|x^{1 / 2}-s_{n}\right|+s_{n}^{1 / 3}\right)^{-p / 4} \in A_{p}(0, \infty) \text { uniformly. }
$$

Proof.

Let us prove (11); the proof of (10) is similar. Making the change of variable $x=s_{n}^{2} z$ in the $A_{p}$ definition, it is easy to show that (11) is equivalent to proving

$$
z^{\alpha-\alpha p / 2-p / 8}\left(\left|z^{1 / 2}-1\right|+s_{n}^{-2 / 3}\right)^{-p / 4} \in A_{p}(0, \infty) \text { uniformly }
$$

Taking into account the behaviour of this expression on the intervals $(0,1 / 2),(1 / 2,3 / 2)$ and $(3 / 2, \infty)$, it is not difficult to check that we only need to see

$$
z^{\alpha-\alpha p / 2-p / 8} \in A_{p}\left(0, \frac{1}{2}\right), \quad\left(\left|z^{1 / 2}-1\right|+s_{n}^{-2 / 3}\right)^{-p / 4} \in A_{p}\left(\frac{1}{2}, \frac{3}{2}\right), \quad z^{\alpha-\alpha p / 2-p / 4} \in A_{p}\left(\frac{3}{2}, \infty\right) .
$$

The first and the third ones are true by (8). Finally, the second one follows from (9) by using $\left|z^{1 / 2}-1\right| \sim|z-1|$ and making a change of variable.

Theorem 1. Let $1<p<\infty$ and $\alpha \geq-1 / 2$. Then, there exists a constant $C$ independent of $n$ and $f$, such that

$$
\left\|S_{n} f\right\|_{L^{p}\left(x^{\alpha}\right)} \leq C\|f\|_{L^{p}\left(x^{\alpha}\right)} \quad \forall f \in L^{p}\left(x^{\alpha}\right)
$$

if and only if $\max \left\{\frac{4}{3}, p_{0}\right\}<p<\min \left\{4, p_{1}\right\}$.

Proof.

To find necessary conditions for (12) we apply the standard argument used for the first time in [12]. The uniform boundedness implies that of the operator $T_{n}=S_{n}-S_{n-1}$,

$$
T_{n}(f, x)=c_{n}(f) j_{n}^{\alpha}(x)=j_{n}^{\alpha}(x) \int_{0}^{\infty} f(t) j_{n}^{\alpha}(t) t^{\alpha} d t
$$

Thus, by using duality we can easily obtain that (12) implies

$$
\left\|j_{n}^{\alpha}\right\|_{L^{p}\left(x^{\alpha}\right)}\left\|j_{n}^{\alpha}\right\|_{L^{q}\left(x^{\alpha}\right)} \leq C .
$$


Taking $n=0$ and applying (5) and (1), we find that $p_{0}<p<p_{1}$. Now, provided that $p_{0}<p<p_{1}$, asymptotic estimates for $J_{\alpha+2 n+1}(x)$ with $n$ and $x$ large enough allow us to show that

$$
\left\|j_{n}^{\alpha}\right\|_{L^{p}\left(x^{\alpha}\right)} \sim \begin{cases}n^{-1-\alpha+2 \alpha / p+2 / p} & \text { if } p<4 \\ n^{-1 / 2-\alpha / 2}(\log n)^{1 / 4} & \text { if } p=4 \\ n^{-5 / 6-\alpha+2 \alpha / p+4 /(3 p)} & \text { if } p>4\end{cases}
$$

This, together with (13), implies $4 / 3<p<4$.

On the other hand, let us suppose that $\max \left\{\frac{4}{3}, p_{0}\right\}<p<\min \left\{4, p_{1}\right\}$ and prove the uniform boundedness of $S_{n} f$.

We need a suitable decomposition of the kernel $K_{n}(x, t)$. In [16] it is proved that

$$
\phi_{\mu}(x, t)=\sum_{k=0}^{\infty} 2(\mu+2 k+1) J_{\mu+2 k+1}(x) J_{\mu+2 k+1}(t)
$$

satisfies

$$
\phi_{\mu}(x, t)=\frac{x t}{x^{2}-t^{2}}\left\{x J_{\mu+1}(x) J_{\mu}(t)-t J_{\mu}(x) J_{\mu+1}(t)\right\} .
$$

Consequently, by (4) and (1) it is clear that

$$
K_{n}(x, t)=\frac{1}{2} x^{-\alpha / 2-1 / 2} t^{-\alpha / 2-1 / 2}\left\{\phi_{\alpha}\left(x^{1 / 2}, t^{1 / 2}\right)-\phi_{\alpha+2 n+2}\left(x^{1 / 2}, t^{1 / 2}\right)\right\} .
$$

Now, by using $z J_{\alpha+2 n+3}(z)=(\alpha+2 n+2) J_{\alpha+2 n+2}(z)-z J_{\alpha+2 n+2}^{\prime}(z)$ we obtain

$$
\phi_{\alpha+2 n+2}(x, t)=\frac{x t}{x^{2}-t^{2}}\left\{J_{\alpha+2 n+2}(x) t J_{\alpha+2 n+2}^{\prime}(t)-J_{\alpha+2 n+2}(t) x J_{\alpha+2 n+2}^{\prime}(x)\right\} .
$$

Hence it follows

$$
\begin{gathered}
K_{n}(x, t)=\frac{x^{-\alpha / 2} t^{-\alpha / 2}}{2(x-t)}\left\{x^{1 / 2} J_{\alpha+1}\left(x^{1 / 2}\right) J_{\alpha}\left(t^{1 / 2}\right)-t^{1 / 2} J_{\alpha}\left(x^{1 / 2}\right) J_{\alpha+1}\left(t^{1 / 2}\right)\right\} \\
+\frac{x^{-\alpha / 2} t^{-\alpha / 2}}{2(x-t)}\left\{x^{1 / 2} J_{\alpha+2 n+2}^{\prime}\left(x^{1 / 2}\right) J_{\alpha+2 n+2}\left(t^{1 / 2}\right)-t^{1 / 2} J_{\alpha+2 n+2}\left(x^{1 / 2}\right) J_{\alpha+2 n+2}^{\prime}\left(t^{1 / 2}\right)\right\} .
\end{gathered}
$$

This enables us to write

$$
S_{n}(f, x)=W_{1}(f, x)-W_{2}(f, x)+W_{3, n}(f, x)-W_{4, n}(f, x),
$$

where

$$
\begin{aligned}
& W_{1}(f, x)=\frac{1}{2} \int_{0}^{\infty} x^{-\alpha / 2+1 / 2} t^{-\alpha / 2} \frac{J_{\alpha+1}\left(x^{1 / 2}\right) J_{\alpha}\left(t^{1 / 2}\right)}{x-t} f(t) t^{\alpha} d t, \\
& W_{2}(f, x)=\frac{1}{2} \int_{0}^{\infty} x^{-\alpha / 2} t^{-\alpha / 2+1 / 2} \frac{J_{\alpha}\left(x^{1 / 2}\right) J_{\alpha+1}\left(t^{1 / 2}\right)}{x-t} f(t) t^{\alpha} d t,
\end{aligned}
$$




$$
\begin{aligned}
& W_{3, n}(f, x)=\frac{1}{2} \int_{0}^{\infty} x^{-\alpha / 2+1 / 2} t^{-\alpha / 2} \frac{J_{\nu}^{\prime}\left(x^{1 / 2}\right) J_{\nu}\left(t^{1 / 2}\right)}{x-t} f(t) t^{\alpha} d t \\
& W_{4, n}(f, x)=\frac{1}{2} \int_{0}^{\infty} x^{-\alpha / 2} t^{-\alpha / 2+1 / 2} \frac{J_{\nu}\left(x^{1 / 2}\right) J_{\nu}^{\prime}\left(t^{1 / 2}\right)}{x-t} f(t) t^{\alpha} d t
\end{aligned}
$$

and $\nu=\alpha+2 n+2$.

Therefore, to prove (12) it is sufficient to see

$$
\left\|W_{i} f\right\|_{L^{p}\left(x^{\alpha}\right)} \leq C\|f\|_{L^{p}\left(x^{\alpha}\right)}, \quad i=1,2
$$

and

$$
\left\|W_{i, n} f\right\|_{L^{p}\left(x^{\alpha}\right)} \leq C\|f\|_{L^{p}\left(x^{\alpha}\right)}, \quad i=3,4 .
$$

In order to apply $A_{p}$ theory we will use the following estimates for Bessel functions and their derivatives:

$$
\begin{gathered}
\left|J_{\nu}(x)\right| \leq C x^{-1 / 4}\left(|x-\nu|+\nu^{1 / 3}\right)^{-1 / 4} \\
\left|J_{\nu}^{\prime}(x)\right| \leq C x^{-3 / 4}\left(|x-\nu|+\nu^{1 / 3}\right)^{1 / 4}
\end{gathered}
$$

where, again, $\nu=\alpha+2 n+2$ and the constant $C$ depends only on $\alpha$. These inequalities can be easily deduced from those used in [1] (see also [15]).

Now, the boundedness of the operators $W_{1}$ and $W_{2}$ is equivalent to

$$
\left\|x^{1 / 2-\alpha / 2} J_{\alpha+1}\left(x^{1 / 2}\right) H\left(t^{\alpha / 2} J_{\alpha}\left(t^{1 / 2}\right) f(t), x\right)\right\|_{L^{p}\left(x^{\alpha}\right)} \leq C\|f(x)\|_{L^{p}\left(x^{\alpha}\right)}
$$

and

$$
\left\|x^{-\alpha / 2} J_{\alpha}\left(x^{1 / 2}\right) H\left(t^{1 / 2+\alpha / 2} J_{\alpha+1}\left(t^{1 / 2}\right) f(t), x\right)\right\|_{L^{p}\left(x^{\alpha}\right)} \leq C\|f(x)\|_{L^{p}\left(x^{\alpha}\right)} .
$$

By using (7) and $x^{\alpha-\alpha p / 2-p / 4} \in A_{p}(0, \infty)$ it follows

$$
\begin{gathered}
\left\|x^{-\alpha / 2} J_{\alpha}\left(x^{1 / 2}\right) H\left(t^{1 / 2+\alpha / 2} J_{\alpha+1}\left(t^{1 / 2}\right) f(t), x\right)\right\|_{L^{p}\left(x^{\alpha}\right)}^{p} \\
\leq C_{1} \int_{0}^{\infty}\left|x^{-\alpha / 2-1 / 4} H\left(t^{1 / 2+\alpha / 2} J_{\alpha+1}\left(t^{1 / 2}\right) f(t), x\right)\right|^{p} x^{\alpha} d x \\
\leq C_{2} \int_{0}^{\infty}\left|x^{1 / 2+\alpha / 2} J_{\alpha+1}\left(x^{1 / 2}\right) f(x)\right|^{p} x^{\alpha-\alpha p / 2-p / 4} d x \\
\leq C_{3} \int_{0}^{\infty}\left|x^{1 / 2+\alpha / 2-1 / 4} f(x)\right|^{p} x^{\alpha-\alpha p / 2-p / 4} d x=C_{3}\|f(x)\|_{L^{p}\left(x^{\alpha}\right)}^{p} .
\end{gathered}
$$

The verification of the other inequality is similar by using (7) and $x^{\alpha-\alpha p / 2+p / 4} \in A_{p}(0, \infty)$. 
To finish the proof we will prove the uniform boundedness of $W_{4, n}$; that of $W_{3, n}$ can be obtained in a similar way. Taking $g(t)=J_{\nu}^{\prime}\left(t^{1 / 2}\right) t^{\alpha / 2+1 / 2} f(t)$ and using consecutively (14), (11) and (15) we have

$$
\begin{gathered}
\left\|W_{4, n} f\right\|_{L^{p}\left(x^{\alpha}\right)}^{p}=2^{-p} \int_{0}^{\infty}\left|\int_{0}^{\infty} x^{-\alpha / 2} t^{\alpha / 2+1 / 2} \frac{J_{\nu}\left(x^{1 / 2}\right) J_{\nu}^{\prime}\left(t^{1 / 2}\right)}{x-t} f(t) d t\right|^{p} x^{\alpha} d x \\
=2^{-p} \int_{0}^{\infty}|H(g, x)|^{p}\left|J_{\nu}\left(x^{1 / 2}\right)\right|^{p} x^{-\alpha p / 2+\alpha} d x \\
\leq C_{1} \int_{0}^{\infty}|H(g, x)|^{p} x^{\alpha-\alpha p / 2-p / 8}\left(\left|x^{1 / 2}-\nu\right|+\nu^{1 / 3}\right)^{-p / 4} d x \\
\leq C_{2} \int_{0}^{\infty}|g(x)|^{p} x^{\alpha-\alpha p / 2-p / 8}\left(\left|x^{1 / 2}-\nu\right|+\nu^{1 / 3}\right)^{-p / 4} d x \leq C_{3}\|f\|_{L^{p}\left(x^{\alpha}\right)}^{p}
\end{gathered}
$$

and the result follows.

\section{$\S 3$. The projection operator $M_{\alpha}$.}

By using (6), the integral operator (2) exists for every $f \in L^{1}\left(x^{\alpha}\right)$ and $\mathcal{H}_{\alpha} f \in L^{\infty}\left(x^{\alpha}\right)$. Actually, we have

$$
\left\|\mathcal{H}_{\alpha} f\right\|_{L^{\infty}\left(x^{\alpha}\right)} \leq \frac{1}{2} C_{\alpha}\|f\|_{L^{1}\left(x^{\alpha}\right)}
$$

However (2) does not exist for $f \in L^{p}\left(x^{\alpha}\right)$ in general and so we will introduce the operator $M_{\alpha}$ in a similar way to that of the disc multiplier.

We consider the space

$$
S^{+}=\left\{f \in C^{\infty}(0, \infty): \forall k, n \geq 0,\left|t^{k} f^{(n)}(t)\right|<C_{k, n}\right\}
$$

with the topology generated by the seminorms $\|\cdot\|_{k, n}, k, n \in \mathbb{N}$, defined by $\|f\|_{k, n}=$ $\sup _{t \in(0, \infty)} t^{k}\left|f^{(n)}(t)\right|$ (see [3] and [4]). It is easy to identify $S^{+}$with the functions $f$ such that $f(t)=\phi(t), t \geq 0$, for some $\phi(t)$ in the Schwartz class $S$.

With this notation $\mathcal{H}_{\alpha}$ is an isomorphism of $S^{+}$onto itself and $\mathcal{H}_{\alpha}^{2}$ is the identity map. Moreover, Fubini's Theorem implies the multiplication formula for the Hankel transform:

$$
\int_{0}^{\infty} \mathcal{H}_{\alpha}(f, x) g(x) x^{\alpha} d x=\int_{0}^{\infty} \mathcal{H}_{\alpha}(g, x) f(x) x^{\alpha} d x, \quad f, g \in S^{+}
$$

Now, taking $g(x)=\mathcal{H}_{\alpha}(f, x)$ and using that $\mathcal{H}_{\alpha}^{2}=\mathrm{Id}$, we have Parseval's formula $\left\|\mathcal{H}_{\alpha} f\right\|_{L^{2}\left(x^{\alpha}\right)}=\|f\|_{L^{2}\left(x^{\alpha}\right)}$.

Since $S^{+}$is dense in $L^{2}\left(x^{\alpha}\right)$, the operator $\mathcal{H}_{\alpha}: S^{+} \rightarrow S^{+}$can be extended to $\mathcal{H}_{\alpha}$ : $L^{2}\left(x^{\alpha}\right) \rightarrow L^{2}\left(x^{\alpha}\right)$ satisfying

$$
\left\|\mathcal{H}_{\alpha} f\right\|_{L^{2}\left(x^{\alpha}\right)}=\|f\|_{L^{2}\left(x^{\alpha}\right)} \quad \text { and } \quad \mathcal{H}_{\alpha}^{2}=\mathrm{Id}
$$


Let $M_{\alpha}$ be the operator defined by

$$
M_{\alpha}(f, x)=\mathcal{H}_{\alpha}\left(\chi_{[0,1]} \mathcal{H}_{\alpha} f, x\right), \quad f \in S^{+}
$$

Note that if $f \in S^{+}$then $\mathcal{H}_{\alpha} f \in S^{+}$; thus $\chi_{[0,1]} \mathcal{H}_{\alpha} f \in L^{2}\left(x^{\alpha}\right)$ and $M_{\alpha} f$ is well defined $\forall f \in S^{+}$.

Theorem 2. Let $\alpha \geq-1 / 2$ and $p_{0}<p<p_{1}$. Then there exists a constant $C_{p, \alpha}$ such that

$$
\left\|M_{\alpha} f\right\|_{L^{p}\left(x^{\alpha}\right)} \leq C_{p, \alpha}\|f\|_{L^{p}\left(x^{\alpha}\right)}, \quad \forall f \in S^{+}
$$

Therefore, $M_{\alpha}$ can be extended to an operator (also denoted $M_{\alpha}$ ) bounded on $L^{p}\left(x^{\alpha}\right)$ such that

i) $\mathcal{H}_{\alpha}\left(M_{\alpha} f\right)=\mathcal{H}_{\alpha}(f) \chi_{[0,1]}$ for all $f \in L^{2}\left(x^{\alpha}\right) \cap L^{p}\left(x^{\alpha}\right)$.

ii) $M_{\alpha}^{2} f=M_{\alpha} f$ for all $f \in L^{p}\left(x^{\alpha}\right)$.

iii) Moreover, for $f \in L^{p}\left(x^{\alpha}\right)$ and $g \in L^{q}\left(x^{\alpha}\right), 1 / p+1 / q=1$, we have

$$
\int_{0}^{\infty} f(x) M_{\alpha}(g, x) x^{\alpha} d x=\int_{0}^{\infty} g(x) M_{\alpha}(f, x) x^{\alpha} d x
$$

Proof.

By using (2) and Fubini's Theorem we obtain

$$
M_{\alpha}(f, x)=\frac{1}{4} \int_{0}^{\infty}\left(\int_{0}^{1} J_{\alpha}(\sqrt{y t}) J_{\alpha}(\sqrt{y x}) d y\right) t^{\alpha / 2} x^{-\alpha / 2} f(t) d t
$$

Moreover, a change of variable in Lommel's formula

$$
\begin{aligned}
\int_{0}^{1} J_{\alpha}(y t) J_{\alpha}(y x) y d y & =\frac{1}{t^{2}-x^{2}}\left(J_{\alpha}(t) x J_{\alpha}^{\prime}(x)-J_{\alpha}(x) t J_{\alpha}^{\prime}(t)\right) \\
& =\frac{1}{t^{2}-x^{2}}\left(t J_{\alpha+1}(t) J_{\alpha}(x)-x J_{\alpha}(t) J_{\alpha+1}(x)\right)
\end{aligned}
$$

(for the last equality, use $\left.z J_{\alpha}^{\prime}(z)=\alpha J_{\alpha}(z)-z J_{\alpha+1}(z)\right)$ leads us to

$$
\begin{aligned}
M_{\alpha}(f, x) & =\frac{1}{2} \int_{0}^{\infty} \frac{t^{1 / 2} J_{\alpha+1}\left(t^{1 / 2}\right) J_{\alpha}\left(x^{1 / 2}\right)-x^{1 / 2} J_{\alpha}\left(t^{1 / 2}\right) J_{\alpha+1}\left(x^{1 / 2}\right)}{t-x} t^{\alpha / 2} x^{-\alpha / 2} f(t) d t \\
& =W_{1}(f, x)-W_{2}(f, x),
\end{aligned}
$$

where $W_{1}$ and $W_{2}$ are bounded operators (see the proof of Theorem 1).

Now, taking into account that $\mathcal{H}_{\alpha}^{2}=\mathrm{Id}$ and using standard density arguments, the statements i) and ii) follow easily. Let us now prove iii): 
From (17), it easily follows that, for $f, g \in L^{2}\left(x^{\alpha}\right)$, we have

$$
\int_{0}^{\infty} \mathcal{H}_{\alpha}(f, x) \mathcal{H}_{\alpha}(g, x) x^{\alpha} d x=\int_{0}^{\infty} f(x) g(x) x^{\alpha} d x
$$

Now, let $U_{1}$ and $U_{2}$ be the bilinear functionals on $L^{p}\left(x^{\alpha}\right) \times L^{q}\left(x^{\alpha}\right)$ defined by

$$
U_{1}(f, g)=\int_{0}^{\infty} f(x) M_{\alpha}(g, x) x^{\alpha} d x
$$

and

$$
U_{2}(f, g)=\int_{0}^{\infty} g(x) M_{\alpha}(f, x) x^{\alpha} d x .
$$

It suffices to show that $U_{1}$ and $U_{2}$ are bounded and coincide on the subset $\left(L^{2}\left(x^{\alpha}\right) \times\right.$ $\left.L^{2}\left(x^{\alpha}\right)\right) \cap\left(L^{p}\left(x^{\alpha}\right) \times L^{q}\left(x^{\alpha}\right)\right)$, which is dense in $L^{p}\left(x^{\alpha}\right) \times L^{q}\left(x^{\alpha}\right)$. The boundedness of $U_{1}$ and $U_{2}$ is clear by Hölder's inequality and $p_{0}<p, q<p_{1}$. Furthermore, by using (19), i) twice, and (19) once again, we have

$$
\begin{gathered}
\int_{0}^{\infty} f(x) M_{\alpha}(g, x) x^{\alpha} d x=\int_{0}^{\infty} \mathcal{H}_{\alpha}(f, x) \mathcal{H}_{\alpha}\left(M_{\alpha} g, x\right) x^{\alpha} d x=\int_{0}^{1} \mathcal{H}_{\alpha}(f, x) \mathcal{H}_{\alpha}(g, x) x^{\alpha} d x \\
=\int_{0}^{\infty} \mathcal{H}_{\alpha}\left(M_{\alpha} f, x\right) \mathcal{H}_{\alpha}(g, x) x^{\alpha} d x=\int_{0}^{\infty} M_{\alpha}(f, x) g(x) x^{\alpha} d x
\end{gathered}
$$

and so the proof is complete.

\section{$\S 4$. Main consequences.}

Definition. Let $\alpha \geq-1 / 2$ and $p_{0}<p<p_{1}$. We define

$$
E_{p, \alpha}=\left\{f \in L^{p}\left(x^{\alpha}\right): M_{\alpha} f=f\right\}
$$

endowed with the topology induced by $L^{p}\left(x^{\alpha}\right)$.

Proposition 1. Let $\alpha \geq-1 / 2, p_{0}<s<r<p_{1}$. Then $E_{s, \alpha} \subset E_{r, \alpha}$ and the inclusion is continuous and dense.

Proof.

From (16) and (18), by using interpolation (see [14]), it follows

$$
\left\|\mathcal{H}_{\alpha} f\right\|_{L^{q}\left(x^{\alpha}\right)} \leq C\|f\|_{L^{p}\left(x^{\alpha}\right)}, \quad 1 \leq p \leq 2 \leq q \leq \infty, \quad \frac{1}{p}+\frac{1}{q}=1
$$

On the other hand, if $p \leq 2$ and $p_{0}<p<p_{1}$, for $f \in S^{+}$we have

$$
\begin{gathered}
\left\|M_{\alpha} f\right\|_{L^{\infty}\left(x^{\alpha}\right)}=\left\|\mathcal{H}_{\alpha}\left(\chi_{[0,1]} \mathcal{H}_{\alpha} f\right)\right\|_{L^{\infty}\left(x^{\alpha}\right)} \\
\leq C_{1}\left\|\chi_{[0,1]} \mathcal{H}_{\alpha} f\right\|_{L^{1}\left(x^{\alpha}\right)} \leq C_{2}\left\|\mathcal{H}_{\alpha} f\right\|_{L^{q}\left(x^{\alpha}\right)} \leq C_{3}\|f\|_{L^{p}\left(x^{\alpha}\right)}
\end{gathered}
$$


By density,

$$
\left\|M_{\alpha} f\right\|_{L^{\infty}\left(x^{\alpha}\right)} \leq C\|f\|_{L^{p}\left(x^{\alpha}\right)} .
$$

Since $M_{\alpha}$ is bounded from $L^{p}\left(x^{\alpha}\right)$ into itself, $p_{0}<p<p_{1}$, by interpolation we obtain $\left\|M_{\alpha} f\right\|_{L^{r}\left(x^{\alpha}\right)} \leq C\|f\|_{L^{p}\left(x^{\alpha}\right)}, r \geq p$. Interpolating one more time, we find

$$
\left\|M_{\alpha} f\right\|_{L^{r}\left(x^{\alpha}\right)} \leq C\|f\|_{L^{s}\left(x^{\alpha}\right)}, \quad p_{0}<s<r<p_{1} .
$$

If $f \in E_{s, \alpha}$ then $M_{\alpha} f=f$ and so (20) leads to $\|f\|_{L^{r}\left(x^{\alpha}\right)} \leq C\|f\|_{L^{s}\left(x^{\alpha}\right)}$, which implies $E_{s, \alpha} \subset E_{r, \alpha}$.

To prove that the inclusion is dense, let $f \in E_{r, \alpha}$. Since $L^{r}\left(x^{\alpha}\right) \cap L^{s}\left(x^{\alpha}\right)$ is dense in $L^{r}\left(x^{\alpha}\right)$, for each $\varepsilon>0$ there exists a function $g \in L^{r}\left(x^{\alpha}\right) \cap L^{s}\left(x^{\alpha}\right)$ such that $\|f-g\|_{L^{r}\left(x^{\alpha}\right)}<$ $\varepsilon$. Taking $h=M_{\alpha} g$ it follows that $h \in E_{p, \alpha}$. Then

$$
\|f-h\|_{L^{r}\left(x^{\alpha}\right)}=\left\|M_{\alpha} f-M_{\alpha} g\right\|_{L^{r}\left(x^{\alpha}\right)} \leq\left\|M_{\alpha}\right\|\|f-g\|_{L^{r}\left(x^{\alpha}\right)}<C \varepsilon
$$

and the proof is complete.

If $1 / p+1 / q=1$, the following result says that the dual space $\left(E_{p, \alpha}\right)^{\prime}$ is isomorphic to $E_{q, \alpha}$ in the standard sense

Proposition 2. Let $\alpha \geq-1 / 2$ and $T$ a bounded linear operator on $E_{p, \alpha}, p_{0}<p<p_{1}$. There exists a unique function $g \in E_{q, \alpha}, 1 / p+1 / q=1$, such that

$$
T(f)=\int_{0}^{\infty} f(x) g(x) x^{\alpha} d x \quad \forall f \in E_{p, \alpha}
$$

Furthermore, $C\|g\|_{L^{q}\left(x^{\alpha}\right)} \leq\|T\| \leq\|g\|_{L^{q}\left(x^{\alpha}\right)}$ for a constant $C>0$ depending only on the norm of $M_{\alpha}$ in $L^{q}\left(x^{\alpha}\right)$.

Proof.

Let $T \in\left(E_{p, \alpha}\right)^{\prime}$. By Hahn-Banach's Theorem, $T$ can be extended to $T \in\left(L^{p}\left(x^{\alpha}\right)\right)^{\prime}$ preserving its norm $\|T\|$. By duality between $L^{p}\left(x^{\alpha}\right)$ and $L^{q}\left(x^{\alpha}\right)$, there exists $h \in L^{q}\left(x^{\alpha}\right)$ such that

$$
T(f)=\int_{0}^{\infty} f(x) h(x) x^{\alpha} d x \quad \forall f \in L^{p}\left(x^{\alpha}\right)
$$

and $\|h\|_{L^{q}\left(x^{\alpha}\right)}=\|T\|$.

If $g=M_{\alpha} h$ from ii) in Theorem 2 it follows $g \in E_{q, \alpha}$. We are going to check that this function satisfies our purposes. If $f \in E_{p, \alpha}$, from iii) in Theorem 2 we have

$$
\begin{gathered}
T(f)=\int_{0}^{\infty} f(x) h(x) x^{\alpha} d x=\int_{0}^{\infty} M_{\alpha}(f, x) h(x) x^{\alpha} d x \\
=\int_{0}^{\infty} M_{\alpha}(h, x) f(x) x^{\alpha} d x=\int_{0}^{\infty} g(x) f(x) x^{\alpha} d x .
\end{gathered}
$$


By using $p_{0}<q<p_{1}$ and Hölder's inequality we have $\|T\| \leq\left\|M_{\alpha} h\right\|_{L^{q}\left(x^{\alpha}\right)}$. Thus, the equivalence of norms $\|g\|_{L^{q}\left(x^{\alpha}\right)} \sim\|T\|$ follows immediately.

To prove the uniqueness, suppose $p \geq 2$ and there exist $g$ and $g^{\prime}$ in $E_{q, \alpha}$ satisfying (21). Then

$$
\int_{0}^{\infty} f(x)\left(g(x)-g^{\prime}(x)\right) x^{\alpha} d x=0 \quad \forall f \in E_{p, \alpha} .
$$

Taking $f=g-g^{\prime} \in E_{q, \alpha} \subset E_{p, \alpha}$ it follows $g-g^{\prime}=0$ a. e. Now, the case $p<2$ is a simple consequence of this considering that $L^{q}\left(x^{\alpha}\right)$ is reflexive and, since $E_{q, \alpha}$ is closed, then $E_{q, \alpha}$ is also reflexive.

As it was pointed out in Introduction, let $B_{p, \alpha}$ stands for the closure in $L^{p}\left(x^{\alpha}\right)$ of the space span $\left\{j_{n}^{\alpha}\right\}_{n=0}^{\infty}$. The following results show the main consequences in this section.

Corollary. Let $\alpha \geq-1 / 2$. Then $\lim _{n \rightarrow \infty}\left\|S_{n} f-f\right\|_{L^{p}\left(x^{\alpha}\right)}=0$ for every $f \in B_{p, \alpha}$ if and only if $\max \left\{\frac{4}{3}, p_{0}\right\}<p<\min \left\{4, p_{1}\right\}$. Moreover, if it is the case, then $B_{p, \alpha}=E_{p, \alpha}$.

Proof.

i) By using (3), (18) and the completeness of the Jacobi system it follows that $\left\{j_{n}^{\alpha}\right\}_{n=0}^{\infty}$ is complete in $E_{2, \alpha}$ and so $B_{2, \alpha}=E_{2, \alpha}$. Hence the convergence for $p=2$ is clear. When $p_{0}<p<p_{1}$ then $j_{n}^{\alpha} \in E_{p, \alpha}$ and therefore $B_{p, \alpha} \subset E_{p, \alpha}$.

If $2<p<p_{1}$ and $f \in E_{p, \alpha}$ it follows that for each $\varepsilon>0$ there exists a function $g \in L^{2}\left(x^{\alpha}\right) \cap L^{p}\left(x^{\alpha}\right)$ such that $\|f-g\|_{L^{p}\left(x^{\alpha}\right)}<\varepsilon$. Taking $h=M_{\alpha} g$ we have $M_{\alpha} h=h$ and so $h \in E_{2, \alpha} \cap E_{p, \alpha}=B_{2, \alpha} \cap E_{p, \alpha}$. Since $M_{\alpha}$ is continuous then $\|f-h\|_{L^{p}\left(x^{\alpha}\right)}=$ $\left\|M_{\alpha} f-M_{\alpha} g\right\|_{L^{p}\left(x^{\alpha}\right)}<C \varepsilon$. As $h \in B_{2, \alpha}$ it follows that there exists $h^{\prime} \in \operatorname{span}\left\{j_{n}^{\alpha}\right\}_{n=0}^{\infty}$ such that $\left\|h-h^{\prime}\right\|_{L^{2}\left(x^{\alpha}\right)}<\varepsilon$. By applying triangle inequality and Proposition 1 we obtain $\left\|f-h^{\prime}\right\|_{L^{p}\left(x^{\alpha}\right)}<C_{1} \varepsilon$ and therefore $E_{p, \alpha} \subset B_{p, \alpha}$. Then the mean convergence for $2<p<$ $\min \left\{4, p_{1}\right\}$ is an immediate consequence of Theorem 1 and Banach-Steinhaus Theorem.

If $\max \left\{\frac{4}{3}, p_{0}\right\}<p<2$, the result is easily obtained by duality.

\section{REFERENCES}

[1] J. A. Barceló and A. Córdoba, Band-limited functions: $L^{p}$-convergence, Trans. Amer. Math. Soc. 313 (1989), 655-669.

[2] A. Córdoba, The disc multiplier, Duke Math. J. 58 (1989), 21-27.

[3] A. J. Durán, Laguerre expansions of tempered distributions and generalized functions, J. Math. Anal. Appl. (to appear).

[4] A. J. Durán, On Hankel transform, Proc. Amer. Math. Soc. 110 (1990), 417-424.

[5] A. Erdélyi et al., "Higher Transcendental Functions," Vol. II, McGraw-Hill, New York, 1953.

[6] A. Erdélyi et al., "Tables of Integral Transforms," Vol. II, McGraw-Hill, New York, 1954.

[7] J. García Cuerva and J. L. Rubio de Francia, "Weighted Norm Inequalities and Related Topics," North-Holland, Amsterdam, 1985.

[8] J. J. Guadalupe, M. Pérez, F. J. Ruiz and J. L. Varona, Mean and weak convergence of Fourier-Bessel series, J. Math. Anal. Appl. (to appear). 
[9] J. J. Guadalupe, M. Pérez and J. L. Varona, Weak behaviour of Fourier-Jacobi series, J. Approx. Theory 61 (1990), 222-238.

[10] J. J. Guadalupe, M. Pérez and J. L. Varona, Mean and weak convergence of some orthogonal Fourier expansions by using $A_{p}$ theory, in Orthogonal Polynomials and Their Applications (Proc. Int. Congr., Laredo/Spain 1987, Ed. J. Vinuesa), 161-169, Lect. Notes Pure Appl. Math., 117, Dekker, New York, 1989.

[11] R. Hunt, B. Muckenhoupt and R. Wheeden, Weighted norm inequalities for the conjugate function and the Hilbert transform, Trans. Amer. Math. Soc. 176 (1973), 227-251.

[12] J. Newman and W. Rudin, Mean convergence of orthogonal series, Proc. Amer. Math. Soc. 3 (1952), 219-222.

[13] H. Pollard, The mean convergence of orthogonal series I, Trans. Amer. Math. Soc. 62 (1947), 387-403.

[14] E. M. Stein and G. Weiss, "Introduction to Fourier Analysis on Euclidean Spaces," Princeton Univ. Press, Princeton, NJ, 1975.

[15] G. N. Watson, "A Treatise on the Theory of Bessel Functions," Cambridge Univ. Press, 1966.

[16] J. E. Wilkins, Jr., Neumann series of Bessel functions, Trans. Amer. Math. Soc. 64 (1948), 359-385.

[17] J. E. Wilkins, Jr., Neumann series of Bessel functions. II, Trans. Amer. Math. Soc. 69 (1950), 55-65.

Juan L. Varona

Departamento de Matemática Aplicada

Colegio Universitario de La Rioja (Universidad de Zaragoza)

Obispo Bustamante, 3

26001 Logroño, SPAIN 